\title{
Outpatient neurosurgery in neuro-oncology
}

\author{
Miguel Marigil, MD, PhD, and Mark Bernstein, MD, MHSc (Bioethics), FRCSC \\ Division of Neurosurgery, Toronto Western Hospital, Toronto, Ontario, Canada
}

\begin{abstract}
Technological breakthroughs along with modern application of awake craniotomy and new neuroanesthesia protocols have led to a progressive development in outpatient brain tumor surgery and improved surgical outcomes. As a result, outpatient neurosurgery has become a standard of care at the authors' center due to its clinical benefits and impact on patient recovery and overall satisfaction. On the other hand, the financial savings derived from its application is also another favorable factor exerting influence on patients, health care systems, and society.

Although validated several years ago and with recent data supporting its application, outpatient brain tumor surgery has not gained the traction that it deserves, based on scientific skepticism and perceived potential for medicolegal issues. The goal of this review, based on the available literature and the senior author's experience in outpatient brain tumor surgery, was to evaluate the most important aspects regarding indications, clinical outcomes, economic burden, and patient perceptions.

https://thejns.org/doi/abs/10.3171/2018.3.FOCUS1831
\end{abstract}

KEYWORDS outpatient neurosurgery; awake craniotomy; early discharge; brain tumor

$\mathrm{N}$ EUROSURGERY has been exponentially evolving during the last 50 years. ${ }^{1,25}$ Development of tools such as neuromonitoring and neuronavigation, along with the implementation of new neuroanesthesia protocols, have helped to shorten surgical times and enhance postoperative care, enabling more rapid recovery and early discharges..$^{30,44,52}$ All of these improvements have led to a redefinition of the primary goal of care in neuro-oncology, with the aim of preserving neurological function and ultimately offering the patient a better quality of life. ${ }^{16,26,31,39,57}$ Consequently, there has been a switch toward less invasive surgeries now, centered on the patient's perceptions and well-being. ${ }^{8,14,31}$

The advent of awake craniotomy allowed neurosurgeons to perform safer maximal resections and decrease postoperative deficits. ${ }^{20,21,29,46,48,50}$ Outpatient brain tumor surgery became part of the neuro-oncology armamentarium after awake craniotomies were introduced and perfected. ${ }^{32}$ This concept was subsequently developed, demonstrating that this technique can be safely applied, with improved surgical outcomes and decreased morbidity compared to craniotomies performed under general anesthesia., ${ }^{74}$ Outpatient or same-day surgery brain tumor resection is defined according to a clearly stablished protocol and inclusion criteria, in which a patient arrives at the hospital on the morning of the procedure and, regardless of the anesthesia technique used, the patient leaves the hospital the same day before 9 PM, after a specific clinical and imaging assessment., ${ }^{72,17,24,53}$ With this system, patients avoid an overnight hospital stay and can start their recuperation outside a health care facility. ${ }^{12}$

The goal of this paper was to review the evolution of outpatient brain tumor resection and evaluate its current applicability in modern neurosurgery.

\section{Outpatient Neurosurgery \\ Awake Craniotomy: Historical Background, Anesthetic Development, and Applicability in Outpatient Setting}

Over decades the main goals in neuro-oncology have been as follows: to optimize the extent of resection and to preserve neurological function and patient's quality of life. ${ }^{37,46-48,50}$ The breakthroughs achieved since the 19th century in the knowledge about cerebral location and cortical mapping, along with the introduction of local anesthetics and sedatives, led to the implementation of modern awake craniotomies. ${ }^{15,29}$ These procedures have their roots in early epilepsy surgery and in the excision of le- 
sions seated in anatomical regions close to eloquent areas within the brain. ${ }^{15}$

Awake craniotomy has been evolving over the last 2 decades. Several factors can be identified as key principles for this evolution: neuroanesthesia development, improvement in microneurosurgical technique and management of intraoperative seizures, and finally the significance of brain plasticity for tumors near eloquent areas. ${ }^{6,11,29,30}$ Regarding neuroanesthesia and surgical techniques, the advent and subsequent implementation of remifentanil plus propofol and/or dexmedetomidine as the anesthetic regimen linked to the current cortical-subcortical mapping and neuronavigation methods have contributed to the success of awake craniotomies. ${ }^{4,6,11,21,29,30,42}$ Interestingly, in their paper published in 2015, Hervey-Jumper et al. ${ }^{29}$ describe the evolution of awake surgery and include a comprehensive analysis of technical recommendations in challenging patients: 2-stage surgery (asleep-awake) for tumors with severe mass effect, introduction of the laryngeal mask to control hypercapnia in obese patients or those with obstructive apnea, iced Ringers solutions to control intraoperative seizures, tailored craniotomies in reoperations, and baseline neurological evaluation before the surgery. Based on their results, the authors illustrate a technique with a high degree of success and low risk profile, with an awake craniotomy failure rate of $0.5 \%$, early (at discharge) postoperative neurological deficit of $9 \%$, and late (more than 3 months after the surgery) neurological deficit rate of $3 \% .^{29}$ Specifically in neuro-oncology, such advances have yielded far-reaching consequences by improving extent of resection, increasing overall survival in low-grade and high-grade gliomas, and decreasing postoperative morbidity. ${ }^{5,20,21,29,46-48,50,51}$ Besides its application in guiding surgery in eloquent areas, awake craniotomy constitutes an invaluable neurosurgical tool by reducing anesthesia risks as well as delivering a direct impact on resource optimization..$^{53}$

As a result of these potential implications, outpatient brain tumor surgery was initially introduced by Bernstein $^{7}$ in 1996, when the senior author started applying this technique in 46 patients prospectively selected to undergo awake craniotomies to evaluate the feasibility of a new same-day brain surgery protocol. The results obtained, with an $89 \%$ successful discharge rate, paved the way for the introduction of this technique.

\section{Impact of Awake Craniotomy on a Patient's Survival and Neurological Morbidity}

The scientific evidence currently available supports the tenet that maximal safe removal of hemispheric low-grade and high-grade gliomas, along with adjuvant therapy, can positively impact a patient's survival. ${ }^{46,50,51}$ However, when tumors are located near an eloquent area, the likelihood of neurological worsening increases as the survival rate decreases. ${ }^{47}$ During the last 2 decades, awake craniotomies have been applied in neuro-oncology, with the aim of maximizing the safety of brain tumor resections. In order to localize functional brain areas, both preoperative (functional neuroimaging, tractography, neuronavigation) and intraoperative techniques have been described. $5,23,38$ Previously published studies have demonstrated the value of cortical and subcortical mapping for decreasing postoperative deficits while improving the extent of resection and overall survival in low- and high-grade gliomas. . $^{18,20,34,46-48,50,51}$ Eventually, a meta-analysis published in 2012 examining the usefulness of intraoperative mapping showed that this technique is associated with a reduction in late (more than 3 months after surgery) postoperative deficits in adult patients with supratentorial infiltrative gliomas, compared to patients in whom brain mapping was not used during surgery; this method assumed a transient increase in temporary deficits within 3 months of surgery. ${ }^{21}$

In conclusion, awake craniotomy with intraoperative mapping of brain functions represents the standard of care in glioma surgery near eloquent areas, because this technique increases the surgical safety and does have a positive impact on neuro-oncological outcomes by increasing the extent of resection and overall survival.

\section{Rationale for Outpatient Brain Tumor Surgery}

There has been a progressively universal trend toward minimizing the physical and psychological impact that surgery can have on oncology patients. Consequently, several factors have switched the inpatient surgery to an outpatient setting, given the fact that early discharges can decrease well-defined risks derived from an in-hospital stay and can have a positive impact on the patient's psychosocial sphere. ${ }^{12,27,49,52}$ Although same-day neurosurgery has proven to be a safe method, with potential benefits for the patient and the health care system globally, its expansion has been faster in other surgical specialties. ${ }^{19,22,43}$

Physicians who perform intracranial neurosurgery have been reluctant to apply same-day surgery protocols, mainly because of the perceived risk of further deterioration when the patient is discharged shortly after an intracranial procedure. Be that as it may, several large-scale series analyzing complications after both biopsy and craniotomy procedures have suitably addressed the time frame after which the physician can consider it safe to discharge a patient from the hospital. ${ }^{10,33,53}$

In their retrospective study performed in 1994 to analyze postsurgical complications after CT-guided stereotactic biopsy in 300 patients, Bernstein and Parrent reported that only 3 of 300 patients developed a neurologically relevant intracranial hemorrhage more than 4 hours after the procedure. ${ }^{9}$ In another study analyzing the incidence and timing of complications after intracranial surgery, Taylor et al. reported clinical deterioration as a consequence of postsurgical hematoma within 6 hours of the procedure in 44 of 2305 patients, and more than 24 hours after the surgery in only 6 patients.$^{54}$ Similarly, in a study of 249 patients who underwent brain biopsies, Kaakaji et al. reported postoperative intracerebral hemorrhage in 8 patients within 6 hours after the procedure. ${ }^{33}$

Therefore, there are 2 factors that can halt an early discharge. The first factor is related to surgical site hematomas, which are acute phenomena that almost always occur within 6 hours of surgery and are responsible for almost immediate patient deterioration. The second factor is a delayed presentation after 24 hours related to edema development around the surgical cavity, which has typically 
been described in up to $10 \%$ of the craniotomies ${ }^{9,52,54}$ but is generally manageable with the rigorous use of dexamethasone.

Supported by these data, Purzner et $a l .{ }^{44}$ published a study with 401 patients prospectively selected to undergo outpatient surgery either for biopsy $(\mathrm{n}=152)$ or for craniotomy $(n=249)$, reporting a $94 \%$ and $93 \%$ success rate of discharge the same day after the procedure in the biopsy and craniotomy group, respectively. Those data are in favor of safe early discharge and do not support any additional benefit from keeping the patient admitted overnight. ${ }^{12,49,56}$

Apart from the positive psychological impact of being in a more familiar environment, avoidance of an overnight stay can prevent the patients from increasing their exposure to hospital-related risks such as nosocomial infections, thromboembolism, and the possibility of being affected by medical errors, which are reported in approximately $7.5 \%$ of overall admissions. ${ }^{3,44}$ Besides, patients' perceptions regarding outpatient surgery have been evaluated by Khu and Bernstein ${ }^{35}$ through qualitative studies, which concluded that this procedure is generally well tolerated by the patient.

\section{Selection of Patients: Inclusion and Exclusion Criteria Protocol}

In order to select properly those patients suitable for outpatient procedures and avoid complications, a clearly defined protocol was initially proposed by Bernstein, ${ }^{7}$ and further validated as a specific checklist, which should be discussed thoroughly with the patient in the preoperative visit. ${ }^{44}$

The inclusion and exclusion criteria used for the Day Surgery Unit (DSU) are summarized in Table 1.

Potentially all patients diagnosed with intraaxial supratentorial tumors who have a close relative or caregiver with whom they can stay the first night near the hospital can benefit from this protocol. ${ }^{12,17}$ On the contrary, some health-related (psychological unsuitability, cases in which duration does not allow for a standard monitoring period of 6 hours, severe pulmonary and/or cardiovascular disease) and other social factors (no access to caregiver or residence far away from hospital) can pose an unacceptable risk to the patient undergoing same-day surgery, and in our view these patients should be admitted. ${ }^{17}$

\section{Proposed Same-Day Surgery Protocol}

1. Key first step: preoperative discussion with surgeon, anesthesiologist, and neuro-oncology nurse practitioner to evaluate the most frequent risks or expected events that the patient can experience.

2. Patient arrives at 5:30 $\mathrm{Am}$ to undergo a preoperative neuronavigation-oriented MRI scan.

3. In the operating room, the neuroanesthesia team sets the intravenous and peripheral lines; arterial and venous central lines are uncommon in this outpatient setting. Prophylactic antibiotics and steroids are administered per protocol, avoiding the use of prophylactic anticonvulsants.

4. Subsequently the patient is placed in the desired position, which fits for the specific location, and the sur-
TABLE 1. Established inclusion/exclusion criteria for outpatient procedures

\begin{tabular}{l}
\hline Inclusion criteria \\
\hline Supratentorial tumor \\
\hline Patient caregiver available \\
\hline $\begin{array}{c}\text { Patient staying relatively close to the hospital (i.e., no more than } \\
1 \text { hr away) }\end{array}$ \\
\hline Exclusion criteria \\
\hline Already an inpatient \\
\hline Significant cardiorespiratory morbidity \\
\hline Airway management concerns \\
\hline Uncontrolled seizures or poor neurological status \\
\hline Anticipated long procedure (>4 hrs) \\
\hline Psychological unsuitability or patient preference \\
\hline
\end{tabular}

gical procedure is performed under the appropriate anesthesia method (awake-local or general intubated). Microneurosurgical technique with image guidance and neurophysiological cortical/subcortical mapping is used to help during the surgery.

5. After the procedure is finished, the patient is transferred to the postanesthesia care unit for 2 hours and then monitored at the DSU for an additional 4 hours.

6. Patients undergo a plain CT scan 4 hours postoperatively and a physician assesses it and their neurological status and determines suitability to discharge.

7. Only patients who adhere to these criteria can be safely discharged. If one of these factors, especially unexpected clinical or imaging abnormalities, is found, the patient is admitted for monitoring.

\section{Clinical Impact of Outpatient Surgery Program}

To our knowledge, there are very few prospectively designed studies in which a brain tumor outpatient protocol has been conducted with success (Table 2). ${ }^{7,10,12,28,44,49}$

The goals for which an outpatient neurosurgery is indicated are as follows: reduction in the risks and complications derived from an in-hospital stay, as well as optimization of health care costs and hospital bed flow. Furthermore, in those cases in which an awake craniotomy with brain mapping is used, which are the majority of outpatient cases, the other main goal is to improve the extent of resection ${ }^{20,21,29}$ while reducing neurological deficits and ultimately improving patients' quality of life..$^{30,45}$

The initial study published by Bernstein in 2001 showed a successful same-day discharge of $89 \%$ in a group of 46 patients, with a complication rate of $11 \%$ and no adverse effects in those patients discharged according to the protocol. ${ }^{7}$ This pioneer feasibility study was followed by a subsequent larger group of patients who were considered suitable to undergo biopsy in an outpatient setting and recapitulated the same rates of successful discharge and complications. ${ }^{10,11,33}$ This Toronto model was adopted successfully in the United Kingdom by Grundy et al., demonstrating the reproducibility of the protocol and confirming its efficacy and safety by implementing it in a different health care system. ${ }^{28}$ 
TABLE 2. Summary of the papers evaluating outpatient brain tumor surgery

\begin{tabular}{|c|c|c|c|c|}
\hline Authors \& Year & Procedure (no. of pts) & $\begin{array}{c}\text { Successful } \\
\text { Discharge Rate }\end{array}$ & Complications & Comments \\
\hline Bernstein, 2001 & Craniotomy (46) & $89 \%$ & $11 \%$ & Pioneer outpatient study \\
\hline Blanshard et al., 2001 & Craniotomy (15) & $88 \%$ & $6 \%$ & $1 \mathrm{pt} \mathrm{had} \mathrm{a} \mathrm{seizure,} 1$ had a headache, $\& 1$ had nausea \\
\hline Kaakaji et al., 2001 & Biopsy (71) & $82 \%$ & $6 \%$ & Among the 13 pts admitted, only 4 were due to complications \\
\hline $\begin{array}{l}\text { Bhardwaj \& Bernstein, } \\
2002\end{array}$ & Biopsy (76) & $97 \%$ & $3 \%$ & $\begin{array}{l}1 \text { pt had a small intraventricular hemorrhage \& } 1 \text { developed } \\
\text { mild leg weakness }\end{array}$ \\
\hline Grundy et al., 2008 & $\begin{array}{l}\text { Craniotomy (11), biopsy } \\
\text { (30) }\end{array}$ & $\begin{array}{l}82 \% \text { craniotomy, } \\
90 \% \text { biopsy }\end{array}$ & $\begin{array}{l}18 \% \text { craniotomy, } \\
3 \% \text { biopsy }\end{array}$ & $\begin{array}{l}1 \text { pt had transient hemiparesis, } 1 \text { had a seizure, \& } 1 \text { had an } \\
\text { intraprocedural hemorrhage after biopsy }\end{array}$ \\
\hline $\begin{array}{l}\text { Boulton \& Bernstein, } \\
2008\end{array}$ & $\begin{array}{l}\text { Craniotomy (145), biopsy } \\
\text { (117) }\end{array}$ & $\begin{array}{l}94 \% \text { craniotomy, } \\
93 \% \text { biopsy }\end{array}$ & $\begin{array}{l}5 \% \text { craniotomy } \\
\quad \& \text { biopsy }\end{array}$ & No adverse effects in the pts discharged \\
\hline Purzner et al., 2011 & $\begin{array}{l}\text { Craniotomy (249), biopsy } \\
\text { (152) }\end{array}$ & $\begin{array}{l}93 \% \text { craniotomy, } \\
94 \% \text { biopsy }\end{array}$ & $\begin{array}{l}7 \% \text { craniotomy, } \\
6 \% \text { biopsy }\end{array}$ & $\begin{array}{l}1 \text { pt had worsening of basal neurological status, } 1 \text { had nau- } \\
\text { sea/headache, } 2 \text { had seizures \& hemorrhage }\end{array}$ \\
\hline Au et al., 2016 & $\begin{array}{l}\text { Craniotomy under general } \\
\text { anesthesia (44) }\end{array}$ & $86 \%$ & $11 \%$ & $\begin{array}{l}6 \text { pts had failure in protocol due to seizure, aphasia, wound } \\
\text { hemostasis, cognitive impairment, \& new deficit }\end{array}$ \\
\hline
\end{tabular}

$\mathrm{Pt}=$ patient.

Following these initial publications, a larger study was performed by the same group, with a sample of 117 and 145 patients selected prospectively to undergo brain biopsy and craniotomy, respectively. Among these, $95 \%$ of the outpatient craniotomies were performed with the patient awake. In this study $93 \%$ of the outpatient biopsy patients and $94 \%$ within the craniotomy group were discharged successfully from the DSU. In those who required inpatient conversion, hemorrhage with worsening basal neurological status, headache, new seizures, and familial preference were registered as the main cause for admission the same evening of the procedure. ${ }^{12}$

These results were also validated by the same group in 2011, which represents so far the largest prospective study that has validated the safety and importance of same-day discharge for brain tumors. In this paper, Purzner et al. ${ }^{44}$ published a successful outpatient discharge rate of $94 \%$ and $93 \%$ for 152 and 249 patients selected to have biopsy and craniotomy, respectively, on an outpatient basis. No patients experienced a negative outcome as a result of this early discharge. These results have been maintained over the years; the current series of outpatient craniotomy is at 564 cases as of January 2018.

\section{Outpatient Neurosurgery: Beyond Awake Procedures}

The potential application of an outpatient setting is not limited to an awake surgery, but can also be suitable for patients under general anesthesia in cases of anticipated awkward positioning or lengthy procedures.

In the series published recently by $\mathrm{Au}$ et al., ${ }^{2}$ it was shown that same-day discharge was planned initially for 44 patients for whom general anesthesia was deemed preferable, with 38 patients $(86 \%)$ successfully completing the protocol. Among the cases requiring inpatient conversion, one was admitted due to wound hemostasis problems, one due to new-onset cognitive impairment, two due to new or worsened weakness, and the other because of a new-onset seizure. These findings parallel the rates noted above for other outpatient studies.

Overall, they confirm that there are no adverse effects as a result of an early discharge in selected patients who undergo outpatient procedures under awake or general anesthesia, and that the same inclusion and exclusion criteria should be applied regardless of the type of anesthesia required.

\section{Patient Satisfaction and Perception}

How patients experience a neurosurgical procedure is one element that is frequently overlooked in patient care. The impact of outpatient brain surgery is made not only on surgical grounds, but also on patients' perception regarding the management of their disease.

Fortunately, a few studies addressing this perspective have been conducted. Interestingly, when this idea was initially presented to 27 patients at the time they were prospectively selected for outpatient surgery, the first response was uncertainty about the possibility of early discharge after brain surgery. ${ }^{36}$ However, as this study conducted in an open-ended interview format revealed, the participants gave positive feedback after they had been educated about the procedure.

Furthermore, approximately half of the patients considered that being at home early helped them in the recovery process. ${ }^{36}$ The positive results regarding outpatient surgical procedures were subsequently reinforced by another group, in which a similar qualitative study designed to evaluate patients' perceptions reproduced the same reassuring feedback in a different health care system in the Western world. ${ }^{58}$

\section{Perioperative Patient Care: Role of Nursing in Outpatient Procedures}

Addressing patients' priorities with perioperative teaching has been shown in an ambulatory setting before ${ }^{13}$ Nurses in this setting help to address and alleviate some of the most common doubts regarding the surgical procedure. This person becomes one of the closest professional contacts with whom the patients can establish a fluent communication about recovery time, expected com- 
plications, medication required, and eventually to manage postoperative concerns. Every outpatient program should implement the presence of this professional as one of the essentials in the protocol.

\section{Financial Balance of Outpatient Neurosurgical Protocols}

Improving surgical outcomes and preserving quality of life are considered as primary outcomes in neuro-oncology. One of the secondary advantages of the outpatient neurosurgery programs is to reduce costs without compromising these primary outcomes. ${ }^{60}$ Very few papers have evaluated the financial impact of outpatient neurosurgery.

One report analyzing the financial differences between outpatient stereotactic biopsy and the cost of spending one night as an inpatient showed savings on the order of Can $\$ 1200.00$ per patient in favor of the early discharge. Another study based in the United States health care system demonstrated that extended observation more than 8 hours after the procedure is not necessary in terms of patient safety, and for some major academic hospitals cannot be financially affordable. ${ }^{33}$

Importantly, one of the largest prospective outpatient spine and brain tumor surgery reports provided reassurance that the outpatient day protocol was a safe and valid surgical alternative in selected patients, with no adverse effect derived from this early discharge. Additionally, it was estimated that a total savings of Can $\$ 1,123,200.00$ could be reached in inpatient-associated costs because the hospital stays are reduced and complications such as nosocomial infections, thromboembolic events, and medical errors are minimized. ${ }^{44}$

These results altogether confirm that outpatient cranial and spine surgery can provide cost savings and be applied regardless of the health care system, without affecting the quality of life or complications. ${ }^{55,56}$ As a result of this decrease in the health care resources that are needed, this format may be a solution in some low-income countries as well..$^{55}$

\section{Other Subspecialties Benefitting From the Outpatient System}

The field of outpatient surgery has been developed at a quicker pace in some specialties other than neurosurgery, such as orthopedics, general surgery, and pain interventions. ${ }^{19,22,43}$ Outpatient surgery, or a setting in which a surgical procedure is performed on a patient who is admitted the morning of the operation and discharged later without having stayed overnight in the hospital, can be applied successfully not only for brain tumors but also in spine operations ${ }^{41,43,44,61}$ and in intra-extracranial endovascular ${ }^{62}$ and open vascular procedures. ${ }^{27}$ The discussion regarding all these details is beyond the scope of this review for brain tumors.

\section{The Future of Outpatient Neurosurgery}

It is true that with the spread in the use of awake craniotomy and the improvement of microneurosurgical techniques linked to superior neuroanesthesia protocols, outpatient intracranial surgery has experienced a revolution. It is important to clarify that there are no randomized trials demonstrating that an outpatient protocol is superior to inpatient surgery in terms of clinical outcomes. However, many different prospective studies performed since 1991 have established its safety and efficacy. Furthermore, qualitative studies have also shown that it is a well-tolerated procedure with a positive impact on patients' disease perception. ${ }^{10,12,36,40,44,56}$

Despite its validation, the protocol has not gained widespread popularity among most of the neurosurgeons devoted to neuro-oncology. In a survey of the members of the American Association of Neurological Surgeons and the Congress of Neurological Surgeons, only 6\% of the physicians performed an outpatient biopsy, even though most of them agreed that discharge on the same day would be safe and an option to reconsider..$^{59}$

On the other hand, outpatient neurosurgery practice on a larger scale poses some potential additional medicolegal and ethical issues, which can contribute to a surgeon's hesitation. In the Western world this outpatient intracranial setting may increase the risk of litigations; for example, in cases in which some anesthetists might prefer not to perform these procedures without a central line or might even keep the patient in a step-down unit for more than 12 hours..$^{55}$ We presume that these factors may be responsible for the lack of reliance on outpatient brain surgery. However, this review has amply demonstrated a safety and efficacy profile that has been successfully and consistently maintained for more than 20 years now. Therefore, we would recommend that more neurosurgeons embrace these procedures in the future.

\section{Conclusions}

Outpatient neurosurgery preserves patients' safety and may provide clinical and psychological benefits. This approach represents an advance in modern minimally invasive surgery without compromising patient care, while at the same time it can provide potential financial savings for both high- and low-income countries.

The safety of outpatient brain tumor surgery has been demonstrated. Nevertheless, it is important to underline the fact that by performing outpatient neurosurgery, not only is there no increase in surgical morbidity as a result of an early discharge, but also almost certainly outpatient surgery contributes to the minimization of patients' exposure to nosocomial infections, medical errors, and thromboembolic complications. On the other hand, it decreases the unnecessary use of health care resources and improves inpatient bed occupation. As a result, it may be appropriate for introduction in the developing world. Hopefully this paper will help to underline the role of outpatient brain tumor surgery and enhance the potential for wider future applicability.

\section{References}

1. Apuzzo ML, Elder JB, Liu CY: The metamorphosis of neurological surgery and the reinvention of the neurosurgeon. Neurosurgery 64:788-795, 2009

2. Au K, Bharadwaj S, Venkatraghavan L, Bernstein M: Outpatient brain tumor craniotomy under general anesthesia. J Neurosurg 125:1130-1135, 2016 
3. Baker GR, Norton PG, Flintoft V, Blais R, Brown A, Cox J, et al: The Canadian Adverse Events Study: the incidence of adverse events among hospital patients in Canada. CMAJ 170:1678-1686, 2004

4. Bekker AY, Kaufman B, Samir H, Doyle W: The use of dexmedetomidine infusion for awake craniotomy. Anesth Analg 92:1251-1253, 2001

5. Berger MS, Hadjipanayis CG: Surgery of intrinsic cerebral tumors. Neurosurgery 61 (1 Suppl):279-305, 2007

6. Berkenstadt H, Perel A, Hadani M, Unofrievich I, Ram Z: Monitored anesthesia care using remifentanil and propofol for awake craniotomy. J Neurosurg Anesthesiol 13:246249, 2001

7. Bernstein M: Outpatient craniotomy for brain tumor: a pilot feasibility study in 46 patients. Can J Neurol Sci 28:120124,2001

8. Bernstein M, Bampoe J: Surgical innovation or surgical evolution: an ethical and practical guide to handling novel neurosurgical procedures. J Neurosurg 100:2-7, 2004

9. Bernstein M, Parrent AG: Complications of CT-guided stereotactic biopsy of intra-axial brain lesions. J Neurosurg 81:165-168, 1994

10. Bhardwaj RD, Bernstein M: Prospective feasibility study of outpatient stereotactic brain lesion biopsy. Neurosurgery 51:358-364, 2002

11. Blanshard HJ, Chung F, Manninen PH, Taylor MD, Bernstein M: Awake craniotomy for removal of intracranial tumor: considerations for early discharge. Anesth Analg 92:89-94, 2001

12. Boulton M, Bernstein M: Outpatient brain tumor surgery: innovation in surgical neurooncology. J Neurosurg 108:649654, 2008

13. Brumfield VC, Kee CC, Johnson JY: Preoperative patient teaching in ambulatory surgery settings. AORN J 64:941946, 948, 951-942, 1996

14. Budrukkar A, Jalali R, Dutta D, Sarin R, Devlekar R, Parab $\mathrm{S}$, et al: Prospective assessment of quality of life in adult patients with primary brain tumors in routine neurooncology practice. J Neurooncol 95:413-419, 2009

15. Bulsara KR, Johnson J, Villavicencio AT: Improvements in brain tumor surgery: the modern history of awake craniotomies. Neurosurg Focus 18(4):e5, 2005

16. Campanella F, Palese A, Del Missier F, Moreale R, Ius T, Shallice T, et al: Long-term cognitive functioning and psychological well-being in surgically treated patients with lowgrade glioma. World Neurosurg 103:799-808, 808.e1-808. e9, 2017

17. Carrabba G, Venkatraghavan L, Bernstein M: Day surgery awake craniotomy for removing brain tumours: technical note describing a simple protocol. Minim Invasive Neurosurg 51:208-210, 2008

18. Chang EF, Clark A, Smith JS, Polley MY, Chang SM, Barbaro NM, et al: Functional mapping-guided resection of low-grade gliomas in eloquent areas of the brain: improvement of long-term survival. Clinical article. J Neurosurg 114:566-573, 2011

19. Curet MJ, Contreras M, Weber DM, Albrecht R: Laparoscopic cholecystectomy. Surg Endosc 16:453-457, 2002

20. De Benedictis A, Moritz-Gasser S, Duffau H: Awake mapping optimizes the extent of resection for low-grade gliomas in eloquent areas. Neurosurgery 66:1074-1084, 2010

21. De Witt Hamer PC, Robles SG, Zwinderman AH, Duffau H, Berger MS: Impact of intraoperative stimulation brain mapping on glioma surgery outcome: a meta-analysis. J Clin Oncol 30:2559-2565, 2012

22. Dravet F, Belloin J, Dupré PF, François T, Robard S, Theard $\mathrm{JL}$, et al: [Role of outpatient surgery in breast surgery. Prospective feasibility study.] Ann Chir 125:668-676, 2000 (Fr)

23. Duffau H: Diffusion tensor imaging is a research and edu- cational tool, but not yet a clinical tool. World Neurosurg 82:e43-e45, 2014

24. Dziedzic T, Bernstein M: Awake craniotomy for brain tumor: indications, technique and benefits. Expert Rev Neurother 14:1405-1415, 2014

25. Elder JB, Hoh DJ, Oh BC, Heller AC, Liu CY, Apuzzo ML: The future of cerebral surgery: a kaleidoscope of opportunities. Neurosurgery 62 (6 Suppl 3):1555-1582, 2008

26. Florman JE, Cushing D, Keller LA, Rughani AI: A protocol for postoperative admission of elective craniotomy patients to a non-ICU or step-down setting. J Neurosurg 127:13921397,2017

27. Goettel N, Chui J, Venkatraghavan L, Tymianski M, Manninen PH: Day surgery craniotomy for unruptured cerebral aneurysms: a single center experience. J Neurosurg Anesthesiol 26:60-64, 2014

28. Grundy PL, Weidmann C, Bernstein M: Day-case neurosurgery for brain tumours: the early United Kingdom experience. Br J Neurosurg 22:360-367, 2008

29. Hervey-Jumper SL, Li J, Lau D, Molinaro AM, Perry DW, Meng L, et al: Awake craniotomy to maximize glioma resection: methods and technical nuances over a 27 -year period. J Neurosurg 123:325-339, 2015

30. Ibrahim GM, Bernstein M: Awake craniotomy for supratentorial gliomas: why, when and how? CNS Oncol 1:71-83, 2012

31. Jalali R, Dutta D: Factors influencing quality of life in adult patients with primary brain tumors. Neuro Oncol 14 (Suppl 4):iv8-iv16, 2012

32. July J, Manninen P, Lai J, Yao Z, Bernstein M: The history of awake craniotomy for brain tumor and its spread into Asia. Surg Neurol 71:621-625, 2009

33. Kaakaji W, Barnett GH, Bernhard D, Warbel A, Valaitis K, Stamp S: Clinical and economic consequences of early discharge of patients following supratentorial stereotactic brain biopsy. J Neurosurg 94:892-898, 2001

34. Keles GE, Lamborn KR, Chang SM, Prados MD, Berger MS: Volume of residual disease as a predictor of outcome in adult patients with recurrent supratentorial glioblastomas multiforme who are undergoing chemotherapy. J Neurosurg 100:41-46, 2004

35. Khu KJ, Bernstein M: Awake craniotomy. J Neurosurg 119: 1645,2013

36. Khu KJ, Doglietto F, Radovanovic I, Taleb F, Mendelsohn D, Zadeh G, et al: Patients' perceptions of awake and outpatient craniotomy for brain tumor: a qualitative study. J Neurosurg 112:1056-1060, 2010

37. Kirsch B, Bernstein M: Ethical challenges with awake craniotomy for tumor. Can J Neurol Sci 39:78-82, 2012

38. Krivosheya D, Prabhu SS: Combining functional studies with intraoperative MRI in glioma surgery. Neurosurg Clin $\mathbf{N}$ Am 28:487-497, 2017

39. Lwu S, Edem I, Banton B, Bernstein M, Vescan A, Gentili F, et al: Quality of life after transsphenoidal pituitary surgery: a qualitative study. Acta Neurochir (Wien) 154:1917-1922, 2012

40. Manninen PH, Balki M, Lukitto K, Bernstein M: Patient satisfaction with awake craniotomy for tumor surgery: a comparison of remifentanil and fentanyl in conjunction with propofol. Anesth Analg 102:237-242, 2006

41. McGirt MJ, Godil SS, Asher AL, Parker SL, Devin CJ: Quality analysis of anterior cervical discectomy and fusion in the outpatient versus inpatient setting: analysis of 7288 patients from the NSQIP database. Neurosurg Focus 39(6):E9, 2015

42. Piccioni F, Fanzio M: Management of anesthesia in awake craniotomy. Minerva Anestesiol 74:393-408, 2008

43. Pugely AJ, Martin CT, Gao Y, Mendoza-Lattes SA: Outpatient surgery reduces short-term complications in lumbar discectomy: an analysis of 4310 patients from the ACS-NSQIP database. Spine (Phila Pa 1976) 38:264-271, 2013 
44. Purzner T, Purzner J, Massicotte EM, Bernstein M: Outpatient brain tumor surgery and spinal decompression: a prospective study of 1003 patients. Neurosurgery 69:119-127, 2011

45. Sacko O, Lauwers-Cances V, Brauge D, Sesay M, Brenner A, Roux FE: Awake craniotomy vs surgery under general anesthesia for resection of supratentorial lesions. Neurosurgery 68:1192-1199, 2011

46. Sanai N, Berger MS: Glioma extent of resection and its impact on patient outcome. Neurosurgery 62:753-764, 264266, 2008

47. Sanai N, Martino J, Berger MS: Morbidity profile following aggressive resection of parietal lobe gliomas. J Neurosurg 116:1182-1186, 2012

48. Sanai N, Polley MY, Berger MS: Insular glioma resection: assessment of patient morbidity, survival, and tumor progression. J Neurosurg 112:1-9, 2010

49. Serletis D, Bernstein M: Prospective study of awake craniotomy used routinely and nonselectively for supratentorial tumors. J Neurosurg 107:1-6, 2007

50. Smith JS, Chang EF, Lamborn KR, Chang SM, Prados MD, Cha $S$, et al: Role of extent of resection in the long-term outcome of low-grade hemispheric gliomas. J Clin Oncol 26:1338-1345, 2008

51. Stummer W, Reulen HJ, Meinel T, Pichlmeier U, Schumacher W, Tonn JC, et al: Extent of resection and survival in glioblastoma multiforme: identification of and adjustment for bias. Neurosurgery 62:564-576, 2008

52. Sughrue ME, Bonney PA, Choi L, Teo C: Early discharge after surgery for intra-axial brain tumors. World Neurosurg 84:505-510, 2015

53. Taylor MD, Bernstein M: Awake craniotomy with brain mapping as the routine surgical approach to treating patients with supratentorial intraaxial tumors: a prospective trial of 200 cases. J Neurosurg 90:35-41, 1999

54. Taylor WA, Thomas NW, Wellings JA, Bell BA: Timing of postoperative intracranial hematoma development and implications for the best use of neurosurgical intensive care. $\mathbf{J}$ Neurosurg 82:48-50, 1995
55. Turel MK, Bernstein M: Is outpatient brain tumor surgery feasible in India? Neurol India 64:886-895, 2016

56. Turel MK, Bernstein M: Outpatient neurosurgery. Expert Rev Neurother 16:425-436, 2016

57. Turel MK, Thakar S, Rajshekhar V: Quality of life following surgery for large and giant vestibular schwannomas: a prospective study. J Neurosurg 122:303-311, 2015

58. Wahab SS, Grundy PL, Weidmann C: Patient experience and satisfaction with awake craniotomy for brain tumours. Br J Neurosurg 25:606-613, 2011

59. Warnick RE, Longmore LM, Paul CA, Bode LA: Postoperative management of patients after stereotactic biopsy: results of a survey of the AANS/CNS section on tumors and a single institution study. J Neurooncol 62:289-296, 2003

60. Witiw CD, Nathan V, Bernstein M: Economics, innovation, and quality improvement in neurosurgery. Neurosurg Clin $\mathbf{N}$ Am 26:197-205, viii, 2015

61. Zahrawi F: Microlumbar discectomy. Is it safe as an outpatient procedure? Spine (Phila Pa 1976) 19:1070-1074, 1994

62. Zanaty M, Daou B, Chalouhi N, Starke RM, Samaniego E, Derdeyn C, et al: Same-day discharge after treatment with the Pipeline Embolization Device using monitored anesthesia care. World Neurosurg 96:31-35, 2016

\section{Disclosures}

The authors report no conflict of interest concerning the materials or methods used in this study or the findings specified in this paper.

\section{Author Contributions}

Conception and design: both authors. Drafting the article: Marigil. Critically revising the article: Bernstein. Study supervision: Bernstein.

\section{Correspondence}

Miguel Marigil: Toronto Western Hospital, Toronto, ON, Canada. mmarigilsanchez@gmail.com. 